\title{
PARASITISMO EMAnastrepha sp. (DIPTERA: TEPHRITIDAE) POR Aganaspis pelleranoi (BRÈTHES, 1924) E Dicerataspis sp. (HYMENOPTERA: FIGITIDAE: EUCOILINAE)
}

\author{
Parasitism of Anastrepha sp. (Diptera: Tephritidae) pupae by Aganaspis pelleranoi \\ (BRÈTHES, 1924) and Dicerataspis sp. (Hymenoptera: Figitidae: Eucoilinae)
}

\author{
Renildo Ismael Félix Costa ${ }^{1}$, Cláudio Gonçalves da Silva², Carlos Henrique Marchiori \\ Bruno Barbosa Amaral ${ }^{4}$, Maurício Marques Poletti ${ }^{4}$, Lucas Castro Torres ${ }^{5}$
}

\begin{abstract}
RESUMO
Objetivou-se com este trabalho conhecer as espécies de parasitóides associados às pupas de Anastrepha sp., em cultivos de goiabeira Psidium guajava L. no município de Lavras - MG. Frutos atacados foram depositados em recipientes plásticos, sobre uma camada de areia fina com $5 \mathrm{~cm}$ de espessura, a qual serviu como substrato para a fase de pupa. As pupas foram separadas do substrato pelo método de flutuação, sendo quantificadas e individualizadas em frascos de vidro contendo areia, onde permaneceram até a emergência das moscas adultas ou de seus parasitóides. Foram coletados 190 pupários de Anastrepha sp., dos quais emergiram 38 parasitóides (Hymenoptera: Figitidae), sendo 20 Aganaspis pelleranoi (Brèthes, 1924) e 18 Dicerataspis sp., correspondendo a um parasitismo natural de $20 \%$.
\end{abstract}

Termos para indexeção: Hymenoptera, Figitidae, controle biológico, mosca-das-frutas, parasitóides.

\begin{abstract}
The aim of this work was to search about the parasitoids species associated to Anastrepha sp. pupae on guava Psidium guajava L. groves in Lavras, MG - Brazil. Fruits with signs of infestation, including oviposition punctures, were picked and placed in plastic containers, under a layer of thin sand, which was utilized as environment for the pupae phase. The pupae were individualized on glass vials containing sand and the emerged fruit flies or theirs parasitoids were collected. A total of 190 Anastrepha sp. Pupae was obtained from which emerged 38 parasitoids (Hymenoptera: Figitidae), being 20 of Aganaspis pelleranoi (Brèthes, 1924) and 18 of Dicerataspis sp., corresponding to a natural parasitism of $20 \%$.
\end{abstract}

Index terms: Hymenoptera, Figitidae, biological control, fruit flies, parasitoids.

\section{(Recebido em 9 de fevereiro de 2004 e aprovado em 16 de setembro de 2005)}

\section{INTRODUÇÃo}

A goiabeira Psidium guajava L., planta da família das Mirtáceas, originária das Américas Central e do Sul, encontra-se amplamente distribuída por todas as regiões tropicais e subtropicais do mundo, sendo cultivada em países como Índia, Paquistão, México, Egito, Venezuela, Brasil, África do Sul, Jamaica, Austrália, Quênia e Porto Rico (MAIA et al., 1991). Essa cultura tem apresentado crescente importância econômica não só pelo elevado valor nutritivo, mas também pela excelente aceitação in natura, além das possibilidades de uso industrial. O Brasil é o terceiro maior produtor mundial de goiaba, sendo os principais Estados produtores São Paulo e Pernambuco, que juntos respondem por cerca de $67 \%$ do volume total da produção nacional. O Estado de Minas Gerais também encontra-se numa posição expressiva com uma produção anual em torno de 4.879 toneladas (AGRIANUAL, 2005).

A despeito de sua relevante posição no cenário mundial, a exportação da goiaba brasileira in natura ainda é inexpressiva, fazendo com que a sua comercialização dependa quase que exclusivamente do mercado interno. De acordo com Dória et al. (2004), o principal entrave à exportação desses frutos é a imposição de barreiras quarentenárias pelos países importadores, visando impedir a introdução das mosca-das-frutas Ceratitis capitata (Wiedemann, 1824) e Anastrepha spp. (Diptera:

\footnotetext{
'Engenheiro Agrônomo; Doutor - Professor de Entomologia da Fundação Educacional de Machado/Centro Superior de Ensino e Pesquisa (FEM/CESEP) Av. Dr. Athayde Pereira de Souza, 730 - Machado, MG - 37750-000 - renildoc2000@yahoo.com

'Biólogo; Mestre - Professor de Zoologia de Invertebrados e Biologia Parasitária do Centro de Ciências Agrárias e Ambientais/CCAA - Universidade Federal do Maranhão/UFMA - Campus IV - BR 222, Km 04 - 65.5000-000 - Chapadinha, MA - ziwky@yahoo.com.br

${ }^{3}$ Biólogo; Doutor - Professor do Centro Federal de Educação Tecnológica de Urutaí - BR 153, Km 633 - 75.650-000 - Morrinhos, GO chmarchiori@yahoo.com.br

${ }^{4}$ Discente do Curso de Agronomia - Estagiário no Curso de Entomologia - Universidade Federal de Lavras/UFLA - Departamento de Entomologia Cx. P. 3037 - 37.500-000 - Lavras, MG.

${ }^{5}$ Engenheiro Agrônomo; Doutorando do Curso de Entomologia - Universidade Federal de Lavras/UFLA - Departamento de Entomologia - Cx. P. 3037 37.500-000 - Lavras, MG.
} 
Tephritidae). Desse modo, essas pragas tornam-se importantes, não só pelos danos diretos causados pelas larvas, que alimentam-se no interior dos frutos, mas também por impedir a comercialização de várias espécies frutíferas no mercado externo, como goiaba, melão, manga, carambola, maçã, pêssego, ameixa (GARCIA \& CORSEUIL, 2004; HICKEL \& DUCROQUET, 1993; KOVALESKI et al., 1999; MACHADO et al., 1995; MALAVASI \& ZUCCHI, 2000).

O controle de mosca-das-frutas na cultura da goiabeira tem sido realizado através da aplicação de defensivos agrícolas nos frutos quando estes ainda estão jovens, suspendendo-se as aplicações 30 dias antes da colheita (GALLO et al., 2002). No entanto, devido à grande exigência dos países importadores com relação a ausência de pragas e resíduos químicos, junto à conscientização ambiental de consumidores e produtores brasileiros, o manejo dessa praga tem se aperfeiçoado. Partindo do monitoramento da flutuação populacional utilizando-se armadilhas caça-mosca, outras alternativas de controle dessa praga estão sendo empregadas; destacando-se o ensacamento dos frutos, a liberação do macho estéril e a utilização de parasitóides (GARCIA et al., 2003; MALAVASI \& NASCIMENTO, 2003; UCHOAFERNANDES \& ZUCCHI, 1999).

Dentre os parasitóides empregados no controle biológico dessas pragas, os himenópteros das subfamílias Eucoilinae (Cynipoidea: Figitidae) e Opiinae (Braconidae), compõem o grupo de inimigos naturais mais importantes no controle de larvas de dípteros frugívoros (Tephritidae, Lonchaeidae e Drosophilidae), devido à sua elevada eficiência em condições naturais (GUIMARÃES et al., 2003). Além disso, esses inimigos naturais podem ser encontrados desde o Estado da Flórida (EUA), sendo amplamente distribuídos nos países da América Latina (OVRUSKI et al., 2000).

Realizou-se este trabalho com objetivo de conhecer as principais espécies de parasitóides associados a moscadas-frutas Anastrepha sp. coletadas em goiaba ( $P$. guajava) no município de Lavras, Minas Gerais, Brasil.

\section{MATERIAL E MÉTODOS}

O estudo foi realizado em cultivos de goiabeira, $P$. guajava, situados no Sítio Rio Grande em Lavras (MG) ( $21^{\circ} 14^{\prime} 30^{\prime \prime} \mathrm{S}$ e $\left.45^{\circ} 00^{\prime} 10^{\prime \prime} \mathrm{W}\right)$, cujos frutos têm sido destinados ao consumo in natura e na fabricação de sucos e doces. A área de plantio possuía, aproximadamente, um alqueire, circundada por pastagens e fragmentos de mata nativa, permanecendo isenta de tratos culturais durante o período das coletas.
Vinte armadilhas caça-mosca, confeccionadas a partir de garrafas plásticas com capacidade para dois litros, foram distribuídas na área de cultivo. Em cada recipiente foram depositados cinco frutos de goiabeira sobre uma camada de $5 \mathrm{~cm}$ de areia fina, servindo como substrato para a pupação. Semanalmente, a areia contendo as pupas da mosca-das-frutas era depositada em baldes com água, onde as pupas eram separadas pelo método de flutuação. Em seguida estas eram retiradas da água com auxílio de uma peneira e postas para secar. Após a secagem, as pupas de cada armadilha eram quantificadas e individualizadas em frascos de vidro contendo areia, mantidos em sala climatizada a $25 \pm 2{ }^{\circ} \mathrm{C}$ até a emergência dos dípteros e/ou de seus parasitóides.

As coletas foram realizadas semanalmente nos meses de maio a julho de 2003. Nesse período a temperatura média foi de $17,5^{\circ} \mathrm{C}$ e a umidade relativa em torno de $70 \%$. A prevalência de parasitismo foi calculada pela fórmula proposta por Bush et al. (1997) e Salles (1995) em que: $\mathrm{P}=$ (pupas parasitadas/total de pupas)x100

\section{RESULTADOS E DISCUSSÃO}

Foram coletados 190 pupários de Anastrepha sp., dos quais emergiram 38 parasitóides pertencentes à família Figitidae, sendo 20 Aganaspis pelleranoi (Brèthes, 1924) e 18 Dicerataspis sp., com uma prevalência de parasitismo natural em torno de $20 \%$ (Tabela 1).

Essa prevalência foi superior àquelas observadas por Marchiori et al. (2000) no município de Itumbiara (GO) e, Silva et al. (2003) na região de Divinópolis (MG), que constataram uma prevalência de parasitismo de $11,2 \%$ e $14,8 \%$, respectivamente. A respeito da menor prevalência de parasitismo, Silva et al. (2003) evidenciaram maior diversidade de parasitóides, obtendo seis espécies distribuídas nas famílias Braconidae, Diapriidae, Figitidae e Pteromalidae, sendo essas diferentes de Dicerataspis sp. e A. pelleranoi registradas nesse estudo.

Estudando os parasitóides associados às moscadas-frutas na região Neotropical, Wharton et al. (1998) concluíram que Dicerataspis spp. são importantes inimigos naturais, especialmente das espécies que constituem a família Drosophilidae. Por outro lado, Guimarães et al. (2003) constataram que $A$. pelleranoi foi a espécie mais abundante no Brasil, representando cerca de 29,9\% de todos os Eucoilinae associados às larvas de moscas frugívoras, se constituindo num importante agente de controle, sobretudo para as espécies da família Tephritidae. Desse modo, o parasitismo ocasionado por A. pelleranoi 
TABELA 1 - Relação de parasitóides (Hymenoptera: Figitidae) coletados em pupários de Anastrepha sp. (Diptera: Tephritidae), em Lavras -MG.

\begin{tabular}{lccc}
\hline \multicolumn{1}{c}{ Espécies } & $\begin{array}{c}\text { No de indivíduos } \\
\text { coletados }\end{array}$ & $\begin{array}{c}\text { Freqüência } \\
(\boldsymbol{\%})\end{array}$ & $\begin{array}{c}\text { Prevalência de } \\
\text { parasitismo }(\boldsymbol{\%})\end{array}$ \\
\hline Aganaspis pelleranoi & 20 & 52,63 & 10,53 \\
Dicerataspis $\mathrm{sp}$. & 18 & 47,37 & 9,47 \\
\hline Total & 38 & 100 & 20 \\
\hline
\end{tabular}

e Dicerataspis sp. em Anastrepha sp. indica que esses inimigos naturais poderão ser utilizados no manejo de mosca- das-frutas em cultivos de goiabeira na região de Lavras (MG).

\section{CONCLUSÕES}

Constatou-se o parasitismo da mosca-das-frutas Anastrepha sp. pelos inimigos naturais A. pelleranoi e Dicerataspis sp., em cultivos de goiabeira na região de Lavras, MG,

A prevalência de parasitismo natural foi de $20 \%$, sendo $10,53 \%$ por $A$. pelleranoi e $9,47 \%$ por Dicerataspis sp.

\section{REFERÊNCIAS BIBLIOGRÁFICAS}

AGRIANUAL. Anuário da agricultura brasileira. 10. ed. São Paulo: FNP Consultoria \& AgroInformativos, 2004. $520 \mathrm{p}$.

BUSH, A. O.; LAFFERTY, K. D.; LOTZ, J. M. Shostak, parasitology meets ecology on its own terms. Journal of Parasitology, Lancaster, v. 83, p. 575-583, 1997.

DÓRIA, H. O. S.; BORTOLI, S. A. de; ALBERGARIA, N. M. M. S. de. Influência do tratamento térmico na eliminação de Ceratitis capitata em frutos de goiaba (Psidium guajava L.). Acta Scientiarum, Maringá, v. 26, n. 1, p. 107111, 2004.

GALLO, D.; NAKANO, O.; SILVEIRA NETO, S.; CARVALHO, R. P. L.; BAPTISTA, G. C.; BERTI FILHO, E.; PARRA, J. R. P.; ZUCHI, R. A.; ALVES, S. B.; VENDRAMIM, J. D.; MARCHINI, L. C.; LOPES, J. R. S.; OMOTO, C. Entomologia agrícola. Piracicaba: FEALQ, 2002. 920 p.
GARCIA, F. R. M.; CAMPOS, J. V.; CORSEUIL, E. Flutuação populacional de Anastrepha fraterculus (Wiedemann, 1830) (Diptera, Tephritidae) na Região Oeste de Santa Catarina, Brasil. Revista Brasileira de Entomologia, São Paulo, v. 47, n. 3, 2003.

GARCIA, F. R. M.; CORSEUIL, E. Native hymenopteran parasitoids associated with fruit flies (Diptera: Tephritidae) in Santa Catarina State, Brazil. Florida Entomologist, Florida, v. 87, n. 4, p. 517-521, 2004.

GUIMARÃES, J. A.; DIAZ, N. B.; GALLARDO, F. E.; ZUCCHI, R. A. Eucoilinae species (Hymenoptera: Cynipoidea: Figitidae) parasitoids of fruit-infesting dipterous larvae in Brazil: identify, geographical distriguition and host associations. Zootaxa, [S.1.], v. 278, p. 1-23, 2003.

HICKEL, E. R.; DUCROQUET, J. P. H. Flutuação populacional de espécies de Anastrepha sp. (Diptera: Tephritidae) relacionadas com a fenologia de frutificação do pêssego e ameixa em Santa Catarina. Anais da Sociedade Entomológica do Brasil, Jaboticabal, v. 22, p. 591-596, 1993.

KOVALESKI, A.; URAMOTO, K.; SUGAYAMA, R. L.; CANAL, D. N. A.; MALAVASI, A. A survey of Anastrepha Schiner (Diptera: Tephritidae) species in the apple growing área of the state of Rio Grande do Sul, Brasil. Revista Brasileira de Entomologia, São Paulo, v. 43, p. 229-234, 1999.

MACHADO, A. E.; SALLES, L. A. B.; LOECK, A. E. Exigências térmicas de Anastrepha fraterculus (Wied.) e estimativa do número de gerações em Pelotas, RS. Anais da Sociedade Entomológica do Brasil, Jaboticabal, v. 24, p. 573-579, 1995. 
MAIA, M. L.; GARCIA, A. E. B.; LEITE, R. S. da S. F. Aspectos econômicos da produção e mercado. In: ITAL. Goiaba: cultura, matéria-prima, processamento e aspectos econômicos. 2. ed. São Paulo, 1991. p. 177-224, 224 p. (Série frutas tropicais, 6).

MALAVASI, A.; NASCIMENTO, A. S. Programa Biofábrica Moscamed Brasil. In: SICONBIOL, 5., 2003, São Pedro. Resumos... São Pedro: [s.n.], 2003. p. 52.

MALAVASI, A.; ZUCCHI, R. A. Moscas-das-frutas de importância econômica no Brasil. São Paulo: Holos, 2000. $327 \mathrm{p}$.

MARCHIORI, C. H.; OLIVEIRA, A. M. S.; MARTINS, F. F.; BOSSI, F. S.; OLIVEIRA, A. T. Espécies de moscas-dasfrutas (Diptera: Tephritidae) e seus parasitóides em Itumbiara-GO. Pesquisa Agropecuária Tropical, Goiânia, v. 30 , p. $73-76,2000$.

OVRUSKI, S.; ALUJA, M.; SIVINSKI, J.; WHARTON, R. Hymenopteran parasitoids on fruit-infesting tephritidae (Diptera) in Latin America and the Southern United States: diversity, distribution, taxonomic status and their use in fruit fly biological control. Integrated
Pest Management Reviews, [S.1.], v. 5, n. 2, p. 81-107, 2000.

SALLES, L. A. B. Estratificação vertical da incidência de Anastrepha fraterculus (Wied.) em fruteiras no sul do Brasil. Anais da Sociedade Entomológica do Brasil, Jaboticabal, v. 24, p. 423-428, 1995.

SILVA, C. G.; MARCHIORI, C. H.; FONSECA, A. R.; TORRES, L. C. Himenópteros parasitóides de larvas de Anastrepha spp. em frutos de carambola (Averrhoa carambola L.) na região de Divinópolis, Minas Gerais, Brasil. Ciência e Agrotecnologia, Lavras, v. 27, n. 6, p. 1264-1267, 2003.

UCHOA-FERNANDES, M. A.; ZUCCHI, R. A. Metodología de colecta de tephritidae y lonchaeidae frugívoros (Díptera: Tephritoidea) y sus parasitoides (Hymenoptera). Anais da Sociedade Entomológica do Brasil, Jaboticabal, v. 28, p. 601-610, 1999.

WHARTON, R. A.; OVRUSKI, S. M.; GILSTRAP, F. E. Neotropical eucoilidae (Cynipoidea) associated with fruitinfesting tephritidae, with new records from Argentina, Bolivia and Costa Rica. Journal of Hymenoptera Research, [S.l.], v. 7, p. 102-115, 1998. 